\title{
Pregnancy Associated Plasma Protein-A and Placental Growth Factor in a Sub-Saharan African Population: A Nested Cross-Sectional Study
}

\author{
Joyce L. Browne ${ }^{1 *}$, Kerstin Klipstein-Grobusch ${ }^{1,2}$, Maria P. H. Koster ${ }^{3}$, \\ Dhivya Ramamoorthy ${ }^{1}$, Edward Antwi ${ }^{1,4}$, Idder Belmouden ${ }^{5}$, Arie Franx ${ }^{3}$, Diederick \\ E. Grobbee ${ }^{1}$, Peter C. J. I. Schielen ${ }^{5}$ \\ 1 Julius Global Health, Julius Center for Health Sciences and Primary Care, University Medical Center \\ Utrecht, Utrecht, the Netherlands, 2 Division of Epidemiology \& Biostatistics, School of Public Health, Faculty \\ of Health Sciences, University of the Witwatersrand, Johannesburg, South Africa, 3 Department of \\ Obstetrics and Gynecology, University Medical Center Utrecht, The Netherlands, 4 Ghana Health Service, \\ Greater Accra Regional Health Directorate, Accra, Ghana, 5 Center for Infectious Diseases Research, \\ Diagnostics and Screening (IDS), National Institute for Public Health and the Environment (RIVM), Bilthoven, \\ The Netherlands \\ *J.L.Browne@umcutrecht.nl
}

\section{Abstract}

\section{Background}

Baseline distributions of pregnancy disorders' biomarkers PIGF and PAPP-A levels are primarily based on Western European populations of Caucasian ethnicity. Differences in PAPP-A and PIGF concentrations by ethnicity have been observed, with increased levels in Afro-Caribbean, East Asian, and South Asian women. Baseline concentrations of subSaharan African women have not been evaluated.

\section{Objectives}

access aticle distributed Creative Commons Attribution License, which permits unrestricted use, distribution, and reproduction in any medium, provided the original author and source are credited.

Data Availability Statement: Data from this study cannot be made publicly available due to an ethical restriction imposed by the Ghana Health Services Ethical Review Committee. However, requests for data can be sent to the authors at (J.L.Browne@umcutrecht.nl).

Funding: PerkinElmer provided PAPP-A and PIGF analysis kits. The sponsor had no role in study design, in the collection, analysis and interpretation of data, in the writing of the report and in the decision to submit the report for publication.
To investigate PIGF and PAPP-A in a sub-Saharan African population and assess the performance of existing reference values of PAPP-A and PIGF.

\section{Methods}

A nested cross-sectional study was conducted in two public hospitals in Accra, Ghana. Out of the original 1010 women enrolled in the cohort, 398 participants were eligible for inclusion with a normotensive singleton gestation and serum samples taken between 56-97 days of pregnancy. PAPP-A and PIGF concentrations were measured with an automated immunoassay. Multiple of the median (MoM) values corrected for gestation and maternal weight for PAPP-A and PIGF were calculated using reference values of a Dutch perinatal screening laboratory based on over 10.000 samples, and PIGF manufacturer reference values, respectively. 
Competing Interests: The authors have declared that no competing interests exist.

\section{Results}

The PAPP-A median MoM was 2.34 (interquartile range (IQR) 1.24-3.97). Median PIGF MoM was 1.25 (IQR 0.95-1.80). Median MoM values for PAPP-A and PIGF tended to be slightly different for various Ghanaian ethnic subgroups.

\section{Conclusions}

PAPP-A and PIGF MoM values appear to be substantially higher in a sub-Saharan African population compared to the Caucasian or Afro-Caribbean MoM values previously reported. The difference suggests the need for a specific correction factor for this population to avoid underestimation of risk for fetal aneuploidies or placental disorders when using PAPP-A and PIGF MoM for screening purposes.

\section{Introduction}

Reduced levels of pregnancy associated plasma protein A (PAPP-A) and placental growth factor (PlGF) around 8 to 14 weeks of gestational age are associated with obstetric complications such as preeclampsia, small-for-gestational age (SGA), intra-uterine growth restriction (IUGR) and stillbirth [1-3]. These markers perform best when combined with others in risk models including maternal characteristics and uterine artery pulsatility index (PI) [2,3]. PAPP-A, together with human chorionic gonadotropin (or its free $\beta$-subunit-free $\beta$-hCG) and ultrasound-obtained fetal nuchal translucency thickness, is used to predict the risk for fetal aneuploidies including Down syndrome [4].

To date, distributions of PlGF and PAPP-A levels in pregnant women are primarily based on Western European populations, predominantly of Caucasian ethnicity. Differences in PAPP-A and PlGF levels by ethnicity have been observed, with increased levels in Afro-Caribbean [5-14], East Asian [5,7-10,12-16], and South Asian [5,7-10,12-14,16] women, though some studies report no difference for Asian women [6,11]. Between studies ethnicity has been diversely defined, limiting study comparability. For Afro-Caribbean women it has been suggested that the difference is not constant, but increasing with gestational age. The mechanisms underlying these differences are poorly understood [6]. Ultimately, not correcting for higher levels in non-Caucasian ethnicities, especially given the known heterogeneity of people from African decent $[17,18]$, could result in a structural underestimation of true risk for fetal aneuploidies or placental disorders $[5,6]$.

Placenta-associated disorders in pregnancy are among the major causes of global maternal and neonatal morbidity and mortality, especially in low resource settings $[19,20]$. Risk prediction in early pregnancy in these settings will allow for efficient resource allocation and targeted prevention strategies including aspirin and calcium supplementation [21,22]. In addition, neonatal morbidity and mortality such as IUGR, SGA and stillbirth can be addressed concomitantly [21]. Antenatal screening programs in most resource limited settings currently focus on a specific number of diseases [23], and the possibility to expand these to predict preventable complications of pregnancy could have a substantial and cost-effective health impact-but will require a further understanding of predictive properties in this population and ethnic differences which should be taken into account.

To the best of our knowledge no study assessed the levels of PlGF and PAPP-A in pregnancy in sub-Saharan African women and compared their concentrations against existing Caucasian 
or Afro-Caribbean reference values. Therefore, the objective of this study is to assess the performance of existing reference values of PAPP-A and PIGF in a sub-Saharan African population in Ghana.

\section{Methods}

\section{Study cohort}

This study was nested in a cohort study of 1010 pregnant adult women and took place in two hospitals in Accra, Ghana (Maamobi General Hospital and Ridge Hospital Outpatient Clinic) between July 2012 and March 2014. Inclusion criteria for the present study were a singleton pregnancy with a gestational age (GA) between 56 and 97 days based on ultrasound at enrolment, maternal age more than 18 years and no known pre-existent hypertension and of whom serum was available for analysis $(\mathrm{n}=403)$. Women in the cohort who had a miscarriage $(\mathrm{n}=39)$, any hypertensive disorder in pregnancy $(\mathrm{n}=91)$, twin pregnancy $(\mathrm{n}=6)$ or fetal aneuploidy based phenotype at delivery, were excluded. Approval to conduct the study was obtained from the Ghana Health Services Ethical Review Committee (GHS-ERC 07/09/11). Written informed consent was obtained from participants prior to study inclusion.

\section{Study procedures}

Participant demographic information including obstetric history was obtained at study inclusion by trained interviewers through structured questionnaires. Serum was obtained at day of study inclusion through venipuncture by a qualified laboratory staff member. After coagulation of the blood, it was centrifuge-spinned within two hours according to hospital protocols, and stored at -20 degrees in a temperature-controlled freezer in the central laboratory of Maamobi hospital. Serum samples obtained at Ridge Regional Hospital were stored in the fridge (4 degrees) and transported daily in a cold box with icepacks to the central laboratory in Maamobi for storage. Serum was shipped on dried-ice to the Netherlands, and stored at $-80^{\circ} \mathrm{C}$ at the Dutch Institute for Public Health and the Environment (RIVM) laboratory until analysis in February 2014.

Obstetric and perinatal outcome information was extracted from the record books at the labor ward and postnatal clinic between $4-8$ weeks postpartum. Data were double entered by two trained research assistants, and accuracy validated by merging of the two datasets and source data consultation when mistyping were flagged.

\section{PAPP-A and PIGF concentration measurement}

Analyses of PAPP-A and PIGF were performed in a single run with a 1235 AutoDELFIA automatic immunoassay system (PerkinElmer, Turku, Finland), using automated dissociationenhanced lanthanide fluorescent immunoassays for serum (PerkinElmer). Summarizing, proteins were eluted in wells pre-coated with anti-PAPP-A and anti-PlGF antibodies in $150 \mu \mathrm{L}$ of buffer for 3 hours. After incubation, diluted serum was aspirated and wells washed six times. PAPP-A and PlGF concentrations were quantified using Europium (Eu) labeled tracer antibodies. After dissociation of tracer antibodies and label and chelation of the Eu ions, the amount of Eu label was measured by excitation with a $340 \mathrm{~nm}$ laser and detection of emitted light at $610 \mathrm{~nm}$, respectively. The limit of quantification for PAPP-A is $0.04 \mathrm{U} / \mathrm{L}$ and for PlGF $7 \mathrm{pg} / \mathrm{ml}$.

A random selection of five samples with a PAPP-A concentration of $>8000 \mathrm{U} / \mathrm{L}$ were reanalyzed to exclude measurement error. Outlier assessment for implausible biomarker 
concentrations ( $>99$ th percentile for PAPP-A MoM $>20$ and PIGF MoM of $>8.25$ ) resulted in exclusion of five samples and serum of 398 participants were included in the analysis.

\section{Statistical analysis}

Demographic and socio-economic and obstetric characteristics of the population were presented as mean and standard deviation (SD) for continuous variables and frequencies and percentages for categorical variables.

Reference equations to calculate gestational age-normalized and maternal weight-corrected PAPP-A multiple of the median (MoM) were obtained from the Dutch national prenatal screening program for Down syndrome, which includes PAPP-A measurements between 57 and 97 days of gestation for of a large population $(>>10000)$ of Dutch women. These were;

PAPP-A:

$$
\begin{aligned}
y & =12605.9606-552.53697 x+7.42649 x^{2}-0.0278 x^{3}, \text { with } x \\
& =\text { gestational age at blood sampling in days }
\end{aligned}
$$

PAPP-A MoM weight correction:

$$
y=\exp (1.23234075-0.0181912 x), \text { with } x=\text { weight in kilograms }
$$

The reference median equation for PlGF was based on a polynominal fit of the reference values for gestational weeks 9 to 13 provided in the kit insert (PerkinElmer). The regression equation for gestation in days was:

$$
y=75.08-1.7769 x+0.01589 x^{2}, \text { with } x=\text { gestational age at blood sampling in days }
$$

As in most populations PlGF concentration is not correlated with maternal weight. Thus, PlGF was not corrected for weight [24].

PAPP-A and PlGF concentrations and MoM values were presented as medians and interquartile ranges (IQR) by gestational week. For PAPP-A, weight-corrected MoM values were calculated also presented.

PAPP-A and PIGF concentrations were scatter plotted against gestational age, and a regression line was calculated using a polynominal fit, after comparison of the best fit between linear and polynominal regression using the R2 statistic. Similarly, individual PAPP-A and PlGF MoM values were plotted against weight. Correlation between PAPP-A and PlGF MoM values was calculated using the $\mathrm{R}$ squared statistics. Regression equations were calculated using the STATA package 'aaplot' [25].

Stratified analyses of PAPP-A and PIGF MoM medians and IQR were conducted for the four major ethnic groups in our population, as self-reported by participants: Akan, Hausa, Ewe and Ga-Dangme. These groups were selected from all ethnicities $(>15)$ based on the availability of at least 30 samples. Given the sample size, difference in medians was qualitatively evaluated.

To assess possibly serum handling effects on the serum biomarker values, clustering of high MoM values by facility or date of sampling was assessed.

Statistical analysis was performed using STATA, version 11.

\section{Results}

Serum PAPP-A and PlGF values were available for 398 women who met the inclusion criteria. Table 1 shows characteristics of included women. The mean age of participants was 28.2 years $( \pm 4.9)$. Mean gestational age at inclusion was 79.4 days $( \pm 11.1)$. The majority of women was 
Table 1. Baseline characteristics of participating pregnant women from Accra, Ghana $(n=398)$.

\begin{tabular}{l|l}
\hline Variable & Mean (sd), range or $\mathbf{N}=(\%)$ \\
\hline Age (years) & $28.2(4.9), 18-43$ \\
\hline Gestational age at booking (days) & $79(11.1), 56-97$ \\
\hline Weight $(\mathrm{kg})$ & $65.3(12.6), 41.6-118.0$ \\
\hline Height $(\mathrm{cm})$ & $161.3(6.0), 142-178$ \\
\hline BMl $(\mathrm{kg} / \mathrm{m} 2)$ & $25.1(4.7), 16.2-41.8$ \\
\hline Parity & \\
\hline Nulliparous & $78(19.6)$ \\
\hline $2-3$ pregnancies & $223(56.0)$ \\
\hline$>4$ pregnancies & $97(24.4)$ \\
\hline BMl & \\
\hline$<18.5$ & $29(7.3)$ \\
\hline $18.5-24.99$ & $185(46.7)$ \\
\hline $25-29.9$ & $124(31.2)$ \\
\hline$>30$ & $59(14.8)$ \\
\hline Ethnicity & \\
\hline Akan & $111(37.9)$ \\
\hline Hausa & $69(17.3)$ \\
\hline Ewe & $82(20.6)$ \\
\hline Ga, Ga-Dangme & $35(8.8)$ \\
\hline Mole, Dagbon, Gonja and others & $61(15.3)$ \\
\hline
\end{tabular}

doi:10.1371/journal.pone.0159592.t001

multiparous (79.4\%). The mean maternal weight was 65.3 kilograms $( \pm 12.6)$, mean height $161.3( \pm 6.0) \mathrm{cm}$. Forty-six percent of participants were overweight or obese (a body mass index (BMI) of 25 or higher). The largest ethnic groups were Akan (37.9\%), followed by Hausa (17.3\%), Ewe (20.6\%) and Ga/Ga-Dangme (8.8\%).

Table 2 presents median PAPP-A and PIGF concentrations and MoM with IQR by gestational week. PAPP-A median concentration at week eight was 565 U/L (IQR 310-980 U/L) and this increased to $8240 \mathrm{U} / \mathrm{L}$ (4080-13000 U/L) at week 13. For PlGF, median concentration at eight weeks was $22.15 \mathrm{pg} / \mathrm{ml}(16.1-31.2 \mathrm{pg} / \mathrm{ml})$, and this increased to $77.7 \mathrm{pg} / \mathrm{ml}(59.2-132 \mathrm{pg} / \mathrm{ml})$ at

Table 2. Median and interquartile range of concentration and MoM of PAPP-A and PIGF by gestational week $(n=398)$.

\begin{tabular}{l|l|l|l|l|l|l}
\hline Weeks & $\mathbf{N}=(\%)$ & $\begin{array}{l}\text { Median concentration } \\
\text { (IQR) PAPP-A U/L }\end{array}$ & $\begin{array}{l}\text { Median } \\
\text { concentration (IQR) } \\
\text { PIGF pg/L }\end{array}$ & $\begin{array}{l}\text { MoM PAPP-A, median } \\
\text { (IQR), adjusted for } \\
\text { gestational age }\end{array}$ & $\begin{array}{l}\text { MoM PAPP-A, median } \\
\text { (IQR), adjusted for } \\
\text { gestational age and weight }\end{array}$ & $\begin{array}{l}\text { MoM PIGF-A, median } \\
\text { (IQR), adjusted for } \\
\text { gestational age }\end{array}$ \\
\hline 8 & $31(7.8)$ & $565(310-980)$ & $22.15(16.1-31.2)^{*}$ & $4.62(1.70-8.3)$ & $4.41(1.57-7.90)$ & \\
\hline 9 & $40(10.1)$ & $972(608-2285)$ & $23.6(19.45-31.4)$ & $2.13(1.32-4.75)$ & $2.52(1.15-4.83)$ & $0.90(0.74-1.20)$ \\
\hline 10 & $80(20.1)$ & $2210(1410-3060)$ & $32.6(25.3-45.0)$ & $2.20(1.45-3.63)$ & $2.10(1.34-3.35)$ & $1.14(0.89-1.58)$ \\
\hline 11 & $73(18.3)$ & $3190(1610-4920)$ & $38.7(32.7-48.6)$ & $1.94(0.98-3.18)$ & $1.97(1.06-3.20)$ & $1.18(0.98-1.48)$ \\
\hline 12 & $75(18.8)$ & $5120(2830-7570)$ & $48.8(35.8-61.9)$ & $2.16(1.17-3.6)$ & $2.25(1.12-3.39)$ & $1.26(0.94-1.63)$ \\
\hline Total & $99(24.9)$ & $8240(4080-13000)$ & $77.7(59.2-132)$ & $2.61(1.36-4.27)$ & $2.46(1.38-3.85)$ & $1.73(1.32-2.94)$ \\
\hline
\end{tabular}

$\mathrm{IQR}=$ interquartile range.

* $n=34$, as statistical outliers could not be excluded as PIGF MoM values were available from week 9 onwards.

Compared to the median MoM values of the reference population (1 by default), the PAPP-A median MoM was 2.30 (1.33-4.14), and 2.34 (1.24-3.97) after weight correction. The median PIGF MoM was 1.25 (0.95-1.80) (Table 3).

doi:10.1371/journal.pone.0159592.t002 
Table 3. MoM values PAPP-A and PIGF for the four main ethnic groups ( $n=398$ for PAPP-A, $n=365$ for PIGF).

\begin{tabular}{l|l|l|l|l}
\hline Ethnicity & $\mathbf{N}=(\%)$ & $\begin{array}{l}\text { MoM PAPP-A, median (IQR), adjusted } \\
\text { for gestational age }\end{array}$ & $\begin{array}{l}\text { MoM PAPP-A, median (IQR), adjusted for } \\
\text { weight and gestational age }\end{array}$ & $\begin{array}{l}\text { Median MoM PIGF (IQR), Adjusted } \\
\text { for gestational age }\end{array}$ \\
\hline Akan & $\begin{array}{l}151 \\
(37.9)\end{array}$ & $2.63(1.67-4.59)$ & $2.50(1.49-4.42)$ & $1.26(0.97-1.73)$ \\
\hline Hausa & $\begin{array}{l}69 \\
(17.3)\end{array}$ & $1.80(1.07-3.18)$ & $2.03(1.04-2.98)$ & $1.15(0.81-1.65)$ \\
\hline Ewe & $\begin{array}{l}82 \\
(20.6)\end{array}$ & $2.50(1.38-5.00)$ & $2.46(1.38-4.45)$ & $1.27(0.94-2.00)$ \\
\hline $\begin{array}{l}\text { Ga, Ga- } \\
\text { Dangme }\end{array}$ & $\begin{array}{l}35 \\
(8.79)\end{array}$ & $2.00(0.82-4.53)$ & $2.07(0.88-4.21)$ & $1.37(0.89-1.73)$ \\
\hline
\end{tabular}

doi:10.1371/journal.pone.0159592.t003

week 13. Both PAPP-A and PlGF concentrations were higher in the Ghanaian population compared to the reference population (Fig 1).

To illustrate the effect of maternal weight on PAPP-A and PIGF distribution in more detail, in Fig 2A PAPP-A MoM and weight-corrected MoM values are presented and Fig 2A PlGF
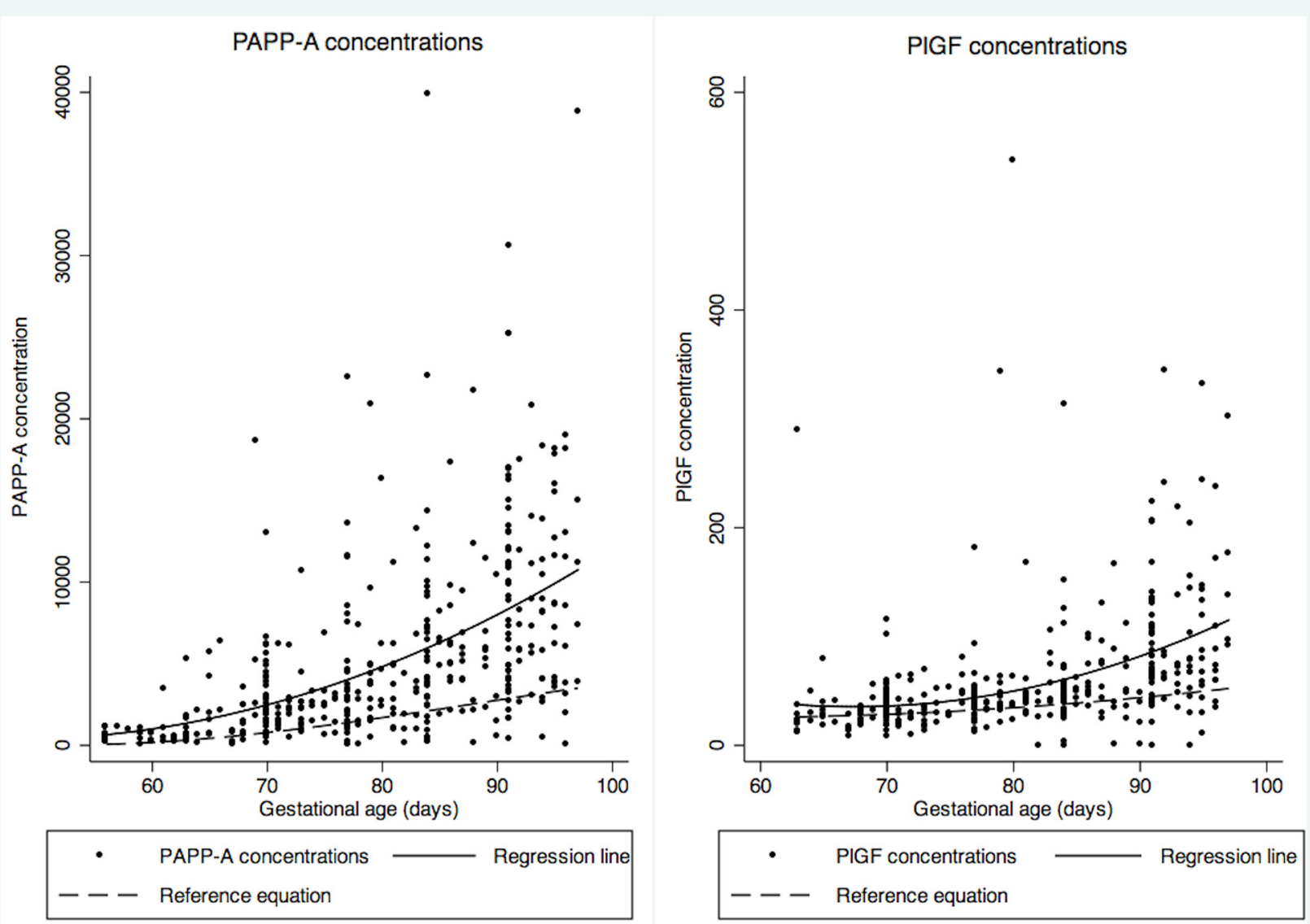

Fig 1. PAPP-A and PIGF concentrations scatterplot, with reference population equation and fitted regression line. Reference population equation PAPP-A: $y=12605.9606-552.53697 x+7.42649 x^{2}-0.0278 x^{3}$; fitted regression line PAPP-A: $y=10123-408.82 x+$ 4.2817 $x^{2}$; Reference population equation PIGF: $y=75.08-1.7769 x+0.01589 x^{2}$; fitted regression line PIGF: $y=448.25-12.247 x=0.908 x^{2}$.

doi:10.1371/journal.pone.0159592.g001 

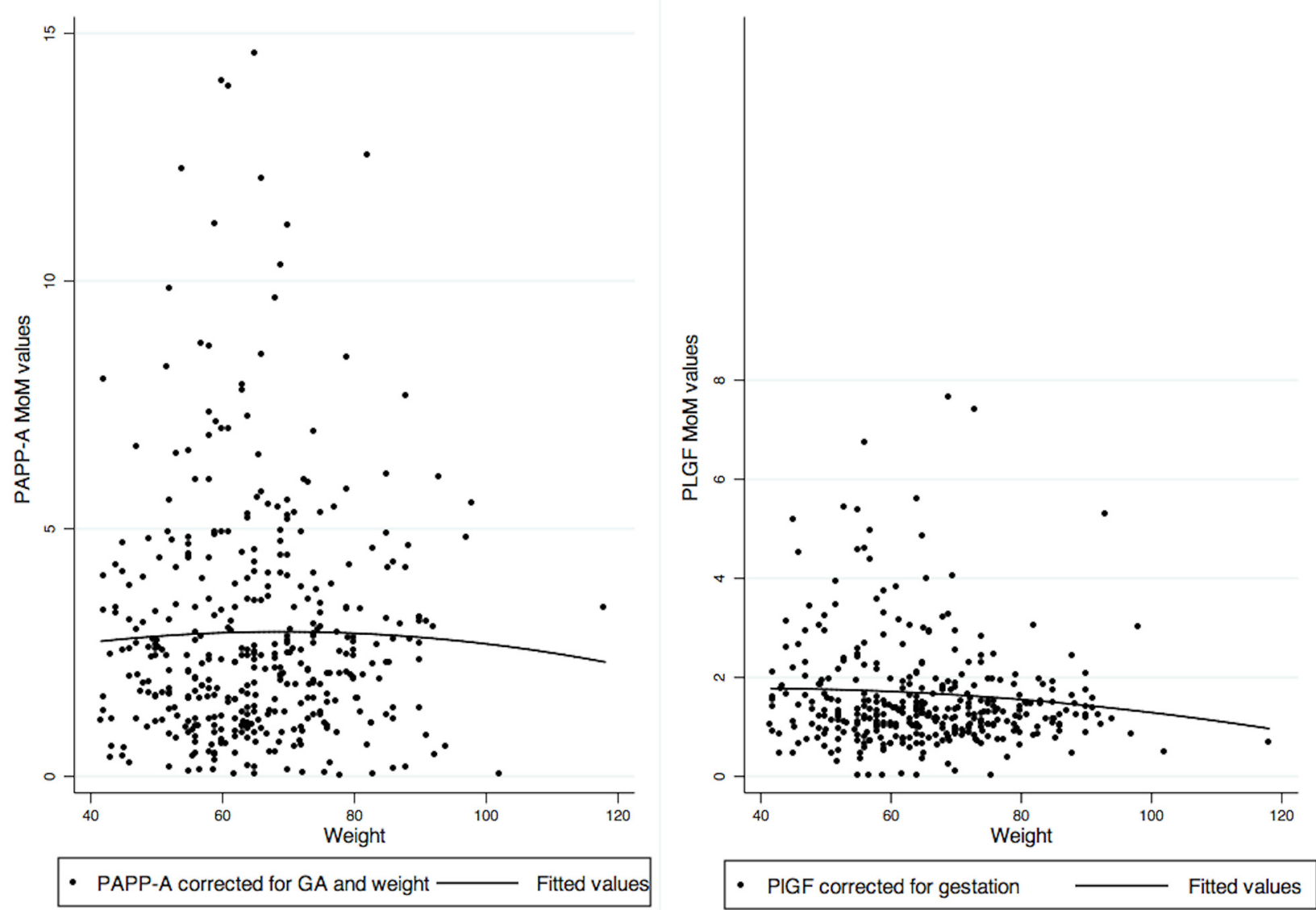

Fig 2. PAPP-A and PIGF MoM values with fitted regression line. PAPP-A regression equation: $y=1.7085+0.03512 x-0.00025 x^{2}$. PIGF: $y=1.6012+0.00938 x-0.0013 x^{2}$.

doi:10.1371/journal.pone.0159592.g002

MoM against weight values are presented. Weight correction resulted in slightly lower MoM values for PAPP-A (Fig 2A). PlGF MoM values remained stable across weights (Fig 2B). Correlation between PAPP-A and PIGF MoM values was low with 0.21 . Clustering by facility or date of sampling was not observed for high MoM values of PlGF or PAPP-A.

For median MoM values for PAPP-A, Hausa women were at the lower end of the spectrum (GA-corrected median MoM 1.80, IQR 1.07-3.18; GA and weight corrected 2.03, IQR 1.04 to 2.98) and Akan women at the higher end (GA-corrected median MoM 2.63, IQR 1.67 to 4.59; GA and weight-corrected median 2.50, IQR 1.49 to 4.42). For PlGF values Hausa women had the lowest MoM values (median MoM 1.15, IQR 0.81 to 1.65) and Ga women were at the higher end of the spectrum (median 1.37, IQR 0.89 to 1.73).

\section{Discussion}

In this study we observed over twice as high median MoM values of PAPP-A and a quarter higher PlGF MoM values in an urban sub-Saharan African population compared to predominantly Caucasian European population.

The PAPP-A median MoM values of our population are higher than previously observed for Afro-Caribbean women (1.46 to 1.70) [5-13]. The PlGF median MoM in our population is similar to that previously reported for Afro-Caribbean women by Padyan et al (1.30), [9] 
though lower than reported by Tsiakkas et al $( \pm 1.55)$ [14]. To exclude the possibility that our observations were a measurement error, we randomly re-analyzed samples with high concentrations, which yielded the same results. The possibility of a delay until freezing due to transportation of samples from Ridge hospital to the main laboratory at Maamobi hospital or handling at Maamobi was assessed, but clustering by facility or date of sampling was not observed for high values. Moreover, given required exposure time to (high) temperatures to induce a significant change in PAPP-A and PlGF concentrations, it is unlikely that this could explain the results [26-28]. Although the preferential rounding for gestational age determination (Fig 1) may affect the MoM when GA is over- or underestimated, this likely occurred randomly and as such would not have affected the median MoM. Therefore, the higher median MoM values in our population compared to previously reported MoM values for Afro-Caribbean women may be a reflection of the heterogeneity of the population from African decent and the differences between Afro-Caribbean population and Black Africans, as discussed by Agyemang et al elsewhere [17]. This may also explain the observed differences in PAPP-A MoM of various ethnic subgroups in our population. The difference in observed MoM values between Afro-Caribbean and Black Africans suggests the need for a specific correction factor for this population for each of the biomarkers, to avoid underestimation in the risk calculation for placental disorders or aneuploidy risk in this population. It also suggests the need to expand the MoM assessments for other sub-Saharan African populations, in order to determine what the variation is within these populations.

Placental differences in weight, volume, surface area and histology have been observed for different ethnicities [29-33], though evidence is inconclusive to determine whether this is a result of biological or environmental factors, and if this underlies the difference in PAPP-A or PlGF concentrations observed. Therefore, future research should include a better understanding of the mechanisms underlying these differences. Possible hypotheses could include higher placental production of these biomarkers, or isoform differences in antibody affinity of antigen presenting sites resulting in higher measurements without difference in absolute concentrations.

\section{Strengths and limitations}

Strengths of this study include the design of this prospective cohort with a representative study population of urban sub-Saharan African women in a lower middle-income country, as public hospitals were included where most women receive maternal care services. The efforts in the design and follow-up are reflected in the high follow-up rate of $83 \%$. To the best of our knowledge, this study is the first to assess MoM reference values in early pregnancy of a sub-Saharan population of PAPP-A and PlGF, as the limited number of studies conducted in sub-Sarahan Africa considered absolute serum concentrations [34-38].

Limitations of this study include the relatively low number of samples $(n=403)$ compared to other ethnicity studies (in high-income country setting) and our ability to control for some known confounders. This, combined with the inherent bias in gestational age determination in early pregnancy reflected in the preferential rounding, may also explain the high median MoM at week 8 , and the absence of a clear upward trend of median PAPP-A MoMs from week 9 to week 13, which is usually observed for PAPP-A. Information about smoking was not available, but unlikely to have affected the results due to the low prevalence among Ghanaian women (0.3\%) [39]. Similarly, although aneuploidies were excluded from the analysis, this was based on clinical assessment and not karyotyping. However, given the low incidence of trisomies $(<1 / 1000$ live births) $[40,41]$, this will likely not have effected the MoM estimates. Information about method of conception including assisted reproductive technique used was also not 
available, but expected to be sporadic at best in the participating low resource public hospitals [42]. As in especially middle-income countries assisted reproductive techniques are increasingly available, the effects of these on pregnancy-related biomarkers will be important to consider in the future [42].

\section{Clinical implications}

Prenatal screening programs for fetal aneuploidies or placental disorders using biomarkers PAPP-A and PlGF are available in most high resources settings, and increasingly available in low- and middle-income countries. Given the global migration patterns and (increasingly) diverse background of pregnant women [43], ethnicity should be considered in planning and execution of prenatal screening programs.

The global burden of maternal and neonatal morbidity and mortality associated with placenta-related disorders such as pre-eclampsia, IUGR and SGA require innovative strategies to optimize antenatal care in resource-constrained settings [19,20,44]. Stratification in antenatal care delivery and targeted interventions for high-risk pregnancies, are a promising approach, but will require the evaluation of predictive properties of in a specific population before implementation.

\section{Conclusion}

PAPP-A and PIGF MoM values appear to be substantially higher in a sub-Saharan African population compared to existing reference values. The difference in observed MoM values between Afro-Caribbean and Black Africans suggests the need for a specific correction factor for this population, to avoid underestimation in the risk calculation for placental disorders and fetal aneuploidy.

\section{Acknowledgments}

We are grateful to all midwives and pregnant women from Ridge Hospital and Maamobi Hospital for their participation in this study. We would like to thank Alidu Wiisibie Huseini and Ernest Addae from Maamobi Hospital laboratory for their support in sample collection. We are appreciative to Henry Kpordzih, Rexford Elorm and Richard Afedi and other research associates for their support.

\section{Author Contributions}

Conceived and designed the experiments: JB PS KKG.

Performed the experiments: PS IB.

Analyzed the data: JB PS KKG MK IB DR DG EA AF.

Wrote the paper: JB PS KKG MK IB DR DG EA AF.

Prepared the first version of the manuscript: JB. Supported this: PS KKG. Commented and approved the final version of the manuscript: JB KKG MK DR EA IB AF DG PS. Accept responsibility for the paper as published: JB KKG MK DR EA IB AF DG PS.

\section{References}

1. Odibo AO, Patel KR, Spitalnik a, Odibo L, Huettner P. Placental pathology, first-trimester biomarkers and adverse pregnancy outcomes. J Perinatol. 2014; 1-6. doi: 10.1038/jp.2013.176 
2. Poon LC, Nicolaides $\mathrm{KH}$. First-trimester maternal factors and biomarker screening for preeclampsia. Prenat Diagn. 2014; 34: 618-27. doi: 10.1002/pd.4397 PMID: 24764257

3. Kuc S, Wortelboer EJ, van Rijn BB, Franx A, Visser GHA, Schielen PCJI. Evaluation of 7 serum biomarkers and uterine artery Doppler ultrasound for first-trimester prediction of preeclampsia: a systematic review. Obstet Gynecol Surv. 2011; 66: 225-239. doi: 10.1097/OGX.0b013e3182227027 PMID: 21756405

4. Nicolaides KH, Spencer K, Avgidou K, Faiola S, Falcon O. Multicenter study of first-trimester screening for trisomy 21 in 75821 pregnancies: results and estimation of the potential impact of individual risk-orientated two-stage first-trimester screening. Ultrasound Obstet Gynecol. 2005; 25: 221-226. doi: 10. 1002/uog.1860 PMID: 15736186

5. Cowans NJ, Spencer K. Effect of gestational age on first trimester maternal serum prenatal screening correction factors for ethnicity and IVF conception. Prenat Diagn. 2013; 33: 56-60. doi: 10.1002/pd. 4010 PMID: 23172419

6. Ball S, Wright D, Sodre D, Lachmann R, Nicolaides $\mathrm{KH}$. Temporal effect of Afro-Caribbean race on serum pregnancy-associated plasma protein-a at 9-13 weeks' gestation in screening for aneuploidies. Fetal Diagn Ther. 2012; 31: 162-9. doi: 10.1159/000336252 PMID: 22377654

7. Wright D, Spencer K, Kagan K K, Tørring N, Petersen OB, Christou A, et al. First-trimester combined screening for trisomy 21 at 7-14 weeks' gestation. Ultrasound Obstet Gynecol. 2010; 36: 404-11. doi: 10.1002/uog.7755 PMID: 20658511

8. Kagan KO, Wright D, Spencer K, Molina FS, Nicolaides KH. First-trimester screening for trisomy 21 by free beta-human chorionic gonadotropin and pregnancy-associated plasma protein-A: impact of maternal and pregnancy characteristics. Ultrasound Obstet Gynecol. 2008; 31: 493-502. doi: 10.1002/uog. 5332 PMID: 18432600

9. Pandya $\mathrm{P}$, Wright $\mathrm{D}$, Syngelaki $\mathrm{A}$, Akolekar R, Nicolaides $\mathrm{KH}$. Maternal serum placental growth factor in prospective screening for aneuploidies at 8-13 weeks' gestation. Fetal Diagn Ther. 2012; 31: 87-93. doi: 10.1159/000335684 PMID: 22286035

10. Spencer K, Heath V, El-Sheikhah A, Ong CYT, Nicolaides KH. Ethnicity and the need for correction of biochemical and ultrasound markers of chromosomal anomalies in the first trimester: a study of Oriental, Asian and Afro-Caribbean populations. Prenat Diagn. 2005; 25: 365-9. doi: 10.1002/pd.1153 PMID: 15906426

11. Ball S, Ekelund C, Wright $D$, Kirkegaard I, Nørgaard $P$, Petersen $O$, et al. Temporal effects of maternal and pregnancy characteristics on serum pregnancy-associated plasma protein- $A$ and free $\beta$-human chorionic gonadotropin at 7-14 weeks' gestation. Ultrasound Obstet Gynecol. 2013; 41: 33-9. doi: 10. 1002/uog.11209 PMID: 22689065

12. Spencer $\mathrm{K}$, Ong CY, Liao AW, Nicolaides $\mathrm{KH}$. The influence of ethnic origin on first trimester biochemical markers of chromosomal abnormalities. Prenat Diagn. 2000; 20: 491-4. PMID: 10861715

13. Wright $D$, Silva $M$, Papadopoulos $S$, Wright $A$, Nicolaides $K H$. Serum pregnancy associated plasma protein-A in the three trimesters of pregnancy: effects of maternal characteristics and medical history. Ultrasound Obstet Gynecol. 2015; doi: 10.1002/uog.14870

14. Tsiakkas A, Duvdevani N, Wright A, Wright D, Nicolaides KH. Serum placental growth factor in the three trimesters of pregnancy: effects of maternal characteristics and medical history. Ultrasound Obstet Gynecol. 2015; 45: 591-8. doi: 10.1002/uog.14811 PMID: 25653039

15. Leung TY, Spencer K, Leung TN, Fung TY, Lau TK. Higher median levels of free beta-hCG and PAPP$A$ in the first trimester of pregnancy in a Chinese ethnic group. Implication for first trimester combined screening for Down's syndrome in the Chinese population. Fetal Diagn Ther. 2006; 21: 140-3. doi: 10. 1159/000089064 PMID: 16354992

16. Manotaya S, Zitzler J, Li X, Wibowo N, Pham TM, Kang MS, et al. Effect of ethnicity on first trimester biomarkers for combined trisomy 21 screening: results from a multicenter study in six Asian countries. Prenat Diagn. 2015; 35: 735-40. doi: 10.1002/pd.4602 PMID: 25858516

17. Agyemang C, Bhopal R, Bruijnzeels M. Negro, Black, Black African, African Caribbean, African American or what? Labelling African origin populations in the health arena in the 21st century. J Epidemiol Community Health. 2005; 59: 1014-8. doi: 10.1136/jech.2005.035964 PMID: 16286485

18. Tishkoff SA, Reed FA, Friedlaender FR, Ehret C, Ranciaro A, Froment A, et al. The genetic structure and history of Africans and African Americans. Science. 2009; 324: 1035-44. doi: 10.1126/science. 1172257 PMID: 19407144

19. Kassebaum NJ, Bertozzi-Villa A, Coggeshall MS, Shackelford KA, Steiner C, Heuton KR, et al. Global, regional, and national levels and causes of maternal mortality during 1990-2013: a systematic analysis for the Global Burden of Disease Study 2013. Lancet. 2014; 384: 980-1004. doi: 10.1016/S0140-6736 (14)60696-6 PMID: 24797575 
20. Lawn JE, Blencowe H, Oza S, You D, Lee ACC, Waiswa P, et al. Every Newborn: progress, priorities, and potential beyond survival. Lancet. 2014; 384: 189-205. doi: 10.1016/S0140-6736(14)60496-7 PMID: 24853593

21. Bujold E, Roberge S, Nicolaides KH. Low-dose aspirin for prevention of adverse outcomes related to abnormal placentation. Prenat Diagn. 2014; 34: 642-8. doi: 10.1002/pd.4403 PMID: 24799357

22. Hofmeyr GJ, Lawrie TA, Atallah AN, Duley L, Torloni MR. Calcium supplementation during pregnancy for preventing hypertensive disorders and related problems. Cochrane database Syst Rev. 2014; 6: CD001059. doi: 10.1002/14651858.CD001059.pub4 PMID: 24960615

23. The Partnership for Maternal Newborn and Child Health. Opportunities for Africa's Newborns [Internet]. Geneva; 2006. Available: http://www.who.int/pmnch/media/publications/oanfullreport.pdf.

24. Kuc S, Koster MPH, Franx A, Schielen PCJI, Visser GHA. Maternal characteristics, mean arterial pressure and serum markers in early prediction of preeclampsia. PLoS One. 2013; 8: e63546. doi: 10.1371/ journal.pone.0063546 PMID: 23717445

25. Cox NJ. AAplot. Durham: StataCorp LP; 2011.

26. Cruz J, Cruz G, Minekawa R, Maiz N, Nicolaides KH. Effect of temperature on free beta-human chorionic gonadotropin and pregnancy-associated plasma protein-A concentration. Ultrasound Obstet Gynecol. 2010; 36: 141-6. doi: 10.1002/uog.7688 PMID: 20503238

27. Cowans NJ, Stamatopoulou A, Hellström J, Mäkelä M-M, Spencer K. PAPP-A and free ss-hCG stability in first trimester serum using PerkinElmer AutoDELFIA and DELFIA Xpress systems. Prenat Diagn. 2010; 30: 127-32. doi: 10.1002/pd.2423 PMID: 20014168

28. Cowans $\mathrm{NJ}$, Alfthan $\mathrm{H}$, Stenman UH, Spencer K. Stability of first trimester placental growth factor in serum and whole blood. Prenat Diagn. 2011; 31: 1193-7. doi: 10.1002/pd.2894 PMID: 22028208

29. Perry IJ, Beevers DG, Whincup PH, Bareford D. Predictors of ratio of placental weight to fetal weight in multiethnic community. BMJ. 1995; 310: 436-9. PMID: 7873949

30. Sivarao S, Vidyadaran MK, Jammal ABE, Zainab S, Goh YM, Ramesh KN. Weight, Volume and Surface Area of Placenta of Normal Pregnant Women and their Relation to Maternal and Neonatal Parameters in Malay, Chinese and Indian Ethnic Groups. Placenta. 2002; 23: 691-696. doi: 10.1053/plac. 2002.0817 PMID: 12361688

31. Williams LA, Evans SF, Newnham JP. Prospective cohort study of factors influencing the relative weights of the placenta and the newborn infant. BMJ. 1997; 314: 1864-8. PMID: 9224128

32. Dombrowski MP. Birth Weight-Length Ratios, Ponderal Indexes, Placental Weights, and Birth WeightPlacenta Ratios in a Large Population. Arch Pediatr Adolesc Med. American Medical Association; 1994; 148: 508. doi: 10.1001/archpedi.1994.02170050066012

33. Jackson MR, Mayhew TM, Haas JD. The volumetric composition of human term placentae: altitudinal, ethnic and sex differences in Bolivia. J Anat. Wiley-Blackwell; 1987; 152: 173-87.

34. Muy-Rivera M, Vadachkoria S, Woelk GB, Quu C, Mahomed K, Williams MA. Maternal plasma VEGF, sVEGF-R1, and PIGF concentrations in preeclamptic and normotensive pregnant Zimbabwean women. Physiol Res. 2005; 54: 611-22. PMID: 15717861

35. Darling AM, McDonald CR, Conroy AL, Hayford KT, Liles WC, Wang M, et al. Angiogenic and inflammatory biomarkers in midpregnancy and small-for-gestational-age outcomes in Tanzania. Am J Obstet Gynecol. 2014; 211: 509.e1-8. doi: 10.1016/j.ajog.2014.05.032

36. Govender N, Naicker T, Moodley J. Maternal imbalance between pro-angiogenic and anti-angiogenic factors in HIV-infected women with pre-eclampsia. Cardiovasc J Afr. 2013; 24: 174-9. doi: 10.5830/ CVJA-2013-029 PMID: 24217170

37. Govender L, Mackraj I, Gathiram P, Moodley J. The role of angiogenic, anti-angiogenic and vasoactive factors in pre-eclamptic African women: early- versus late-onset pre-eclampsia. Cardiovasc J Afr. 2012; 23: 153-9. doi: 10.5830/CVJA-2012-003 PMID: 22555639

38. Powis KM, McElrath TF, Hughes MD, Ogwu A, Souda S, Datwyler SA, et al. High viral load and elevated angiogenic markers associated with increased risk of preeclampsia among women initiating highly active antiretroviral therapy in pregnancy in the Mma Bana study, Botswana. J Acquir Immune Defic Syndr. 2013; 62: 517-24. doi: 10.1097/QAl.0b013e318286d77e PMID: 23344545

39. Owusu-Dabo E, Lewis S, McNeill A, Gilmore A, Britton J. Smoking uptake and prevalence in Ghana. Tob Control. 2009; 18: 365-70. doi: 10.1136/tc.2009.030635 PMID: 19581276

40. Molteno C, Smart R, Viljoen D, Sayed R, Roux A. Twenty-year birth prevalence of Down syndrome in Cape Town, South Africa. Paediatr Perinat Epidemiol. 1997; 11: 428-35. PMID: 9373864

41. Geerts L. Prenatal diagnosis of chromosomal abnormalities in a resource-poor setting. Int $\mathrm{J}$ Gynaecol Obstet. 2008; 103: 16-21. doi: 10.1016/j.ijgo.2008.05.028 PMID: 18708189 
42. Inhorn MC, Patrizio $P$. Infertility around the globe: new thinking on gender, reproductive technologies and global movements in the 21st century. Hum Reprod Update. 2015; doi: 10.1093/humupd/dmv016

43. Gagnon AJ, Redden KL. Reproductive health research of women migrants to Western countries: A systematic review for refining the clinical lens. Best Pract Res Clin Obstet Gynaecol. 2016; 32: 3-14. doi: 10.1016/j.bpobgyn.2016.01.005 PMID: 26925856

44. Bhutta ZA, Das JK, Bahl R, Lawn JE, Salam RA, Paul VK, et al. Can available interventions end preventable deaths in mothers, newborn babies, and stillbirths, and at what cost? Lancet. 2014; 384: 34770. doi: 10.1016/S0140-6736(14)60792-3 PMID: 24853604 